\title{
Mixing Matrix-corrected Whole-body Pharmacokinetic Modeling Using Longitudinal Micro-computed Tomography and Fluorescence-mediated Tomography
}

\author{
Simin Zuo, ${ }^{1}$ Wa'el Al Rawashdeh, ${ }^{2}$ Stefanie Rosenhain, ${ }^{1}$ Zuzanna Magnuska, ${ }^{1}$ \\ Yamoah Grace Gyamfuah, ${ }^{1}$ Fabian Kiessling $\odot{ }^{1,3,4}$ Felix Gremse ${ }^{1,5}$ \\ ${ }^{1}$ Institute for Experimental Molecular Imaging, Helmholtz-Institute for Biomedical Engineering, RWTH Aachen University Clinic, 52074, \\ Aachen, Germany \\ ${ }^{2}$ Miltenyi Biotec GmbH, 51429, Bergisch Gladbach, Germany \\ ${ }^{3}$ Comprehensive Diagnostic Center Aachen (CDCA), 52074, Aachen, Germany \\ ${ }^{4}$ Fraunhofer Institute for Digital Medicine MEVIS, 28359, Bremen, Germany \\ ${ }^{5}$ Gremse-IT GmbH, Dennewartstr. 25, 52068, Aachen, Germany
}

\begin{abstract}
Purpose: Pharmacokinetic modeling can be applied to quantify the kinetics of fluorescently labeled compounds using longitudinal micro-computed tomography and fluorescence-mediated tomography $(\mu \mathrm{CT}-\mathrm{FMT})$. However, fluorescence blurring from neighboring organs or tissues and the vasculature within tissues impede the accuracy in the estimation of kinetic parameters. Contributions of elimination and retention activities of fluorescent probes inside the kidneys and liver can be hard to distinguish by a kinetic model. This study proposes a deconvolution approach using a mixing matrix to model fluorescence contributions to improve whole-body pharmacokinetic modeling.

Procedures: In the kinetic model, a mixing matrix was applied to unmix the fluorescence blurring from neighboring tissues and blood vessels and unmix the fluorescence contributions of elimination and retention in the kidney and liver compartments. Accordingly, the kinetic parameters of the hepatobiliary and renal elimination routes and five major retention sites (the kidneys, liver, bone, spleen, and lung) were investigated in simulations and in an in vivo study. In the latter, the pharmacokinetics of four fluorescently labeled compounds (indocyanine green (ICG), HITC-iodide-microbubbles (MB), Cy7-nanogels (NG), and OsteoSense 750 EX (OS)) were evaluated in BALB/c nude mice.

Results: In the simulations, the corrected modeling resulted in lower relative errors and stronger linear relationships (slopes close to 1) between the estimated and simulated parameters, compared to the uncorrected modeling. For the in vivo study, MB and NG showed significantly higher hepatic retention rates $(P<0.05$ and $P<0.05$, respectively), while OS had smaller renal and hepatic retention rates $(P<0.01$ and $P<0.01$, respectively). Additionally, the bone retention rate of OS was significantly higher $(\mathrm{P}<0.01)$.
\end{abstract}

Correspondence to: Fabian Kiessling; e-mail: fkiessling@ukaachen.de,

Felix Gremse; e-mail: fgremse@ukaachen.de 
Conclusions: The mixing matrix correction improves pharmacokinetic modeling and thus enables a more accurate assessment of the biodistribution of fluorescently labeled pharmaceuticals by $\mu \mathrm{CT}$-FMT.

Key words: Mixing matrix, Pharmacokinetic modeling, Fluorescence-mediated tomography, Computed tomography, Intensity diffusion, Relative blood volume, Elimination routes, Retention sites

\section{Introduction}

Fluorescence-mediated tomography (FMT) is an imaging technique used to assess the three-dimensional distribution of fluorescent probes in preclinical studies [1-3]. In recent years, the sensitivity and accuracy of FMT have notably increased, resulting in improved detection of fluorescent probes in deep tissue regions [4-7]. Therefore, FMT is a promising modality to quantify the whole-body biodistribution of probes in mice [8-11]. In this context, the application of FMT can also help reduce the number of mice required for biomedical and histological examinations in longitudinal studies.

One main limitation of the FMT technique is the lack of accurate and sharp localization of fluorescently labeled compounds in vivo, especially in deep tissue regions $[12,13]$. In previous studies, combining FMT with a high-resolution anatomical imaging modality, such as micro-computed tomography $(\mu \mathrm{CT})$, could substantially enhance its diagnostic quality $[12,14$ 16]. The shape information and organ-specific absorption and scattering maps derived from the $\mu \mathrm{CT}$ scans can be incorporated into FMT reconstruction algorithms, thus improving the quantification and localization of fluorescence signals [6, 7, 14, 17, 18]. Additionally, the organ-specific pharmacokinetic profiles can be extracted by fusing the FMT images and $\mu \mathrm{CT}$-based organ segmentation [17-19].

Pharmacokinetic modeling is used to quantify kinetic behaviors from pharmacokinetic curves [20-25]. Compartmental modeling has been routinely applied to derive kinetic parameters in previous studies, facilitating a better understanding of the information contained in the dynamic in vivo imaging data [26]. In contrast to high-resolution imaging methods like CT and ultrasound, in FMT, the fluorescence is blurred between adjacent organs and tissues due to the lower resolution. Fluorescence of small imaging voxels comparable to the finite spatial resolution of FMT (1-3 mm) influences each other between neighboring tissues [3, 8 , $27,28]$. The limitations of the reconstruction for this illposed problem in FMT also cause blurriness, which could be remarkably improved by the incorporation of CT scans [6].

Additionally, vasculature inside organs also affects the fluorescence measurements of tissue compartments [8]. Furthermore, fluorescence data obtained from the liver and kidneys do not just represent retention kinetics of probes but also contain dynamic information about probe elimination - the liver, for example, consists of hepatocytes for elimination activities and Kupffer cells as retention sites
[29-31]. In pharmacokinetic modeling, the fluorescence contributions of elimination and retention in the kidney and liver compartments should be considered. To reduce these fluorescence blurring and mixing effects, we introduce a mixing matrix into the pharmacokinetic modeling to deconvolute the underlying accurate parameters.

Thus, this study aimed to improve parameter estimation in pharmacokinetic modeling using longitudinal $\mu \mathrm{CT}$-FMT data. For the first time, the mixing matrix was applied to unmix the fluorescence blurring from neighboring tissues and blood vessels and differentiate the elimination and retention sub-compartments in the liver and kidneys. The relevance of the mixing matrix was assessed in simulations and comparatively analyzed using in vivo data.

\section{Materials and Methods}

\section{In Vivo $\mu C T$-FMT Imaging}

All animal experimental procedures were approved by the Governmental Review Committee on Animal Care. Healthy BALB/c nude mice (Charles River Laboratory, Sulzfeld, Germany) were anesthetized to be imaged by hybrid $\mu \mathrm{CT}$-FMT ( $\mu \mathrm{CT}$ : Tomoscope 30s Duo, CT Imaging GmbH, Erlangen, Germany; FMT: FMT 2500, PerkinElmer, Waltham, MA, USA), at $0.25,2,4,8,24,48$, and $72 \mathrm{~h}$ after intravenous injection.

In the animal experiments, four fluorescently labeled compounds (five female mice per group, $2 \mathrm{nmol}$ in $100 \mu \mathrm{l}$ solution per mouse) - indocyanine green (ICG, with a molecular weight of $774.96 \mathrm{~g} / \mathrm{mol}$ ), HITC-iodide-microbubbles (MB, with a diameter of 2-3 $\mu \mathrm{m})$, Cy7-nanogels (NG, with a diameter of $117 \pm 5 \mathrm{~nm}$ ), and OsteoSense $750 \mathrm{EX}$ (OS, with a molecular weight of 1101.1 $\mathrm{g} / \mathrm{mol}$ ) - were chosen to cover various elimination routes and retention sites, as described in detail elsewhere [18]. These fluorescent compounds have well-known but different pharmacokinetic properties. After intravenous administration, ICG is almost exclusively eliminated by the liver with low non-specific retention in cells and tissues. MB and NG are mainly taken up by the Kupffer cells and biodegraded by the liver. The bone-targeting OS is mainly eliminated by the kidneys. All of them are watersoluble and can be fluorescently excited by the 745-nm FMT channel.

The 3D fluorescence images were obtained by FMT reconstruction, which uses $\mu \mathrm{CT}$ information acquired for each mouse and time point (Fig. 1a) [6]. The reconstruction method "corrected" the fluorescence underestimation caused by a rich 

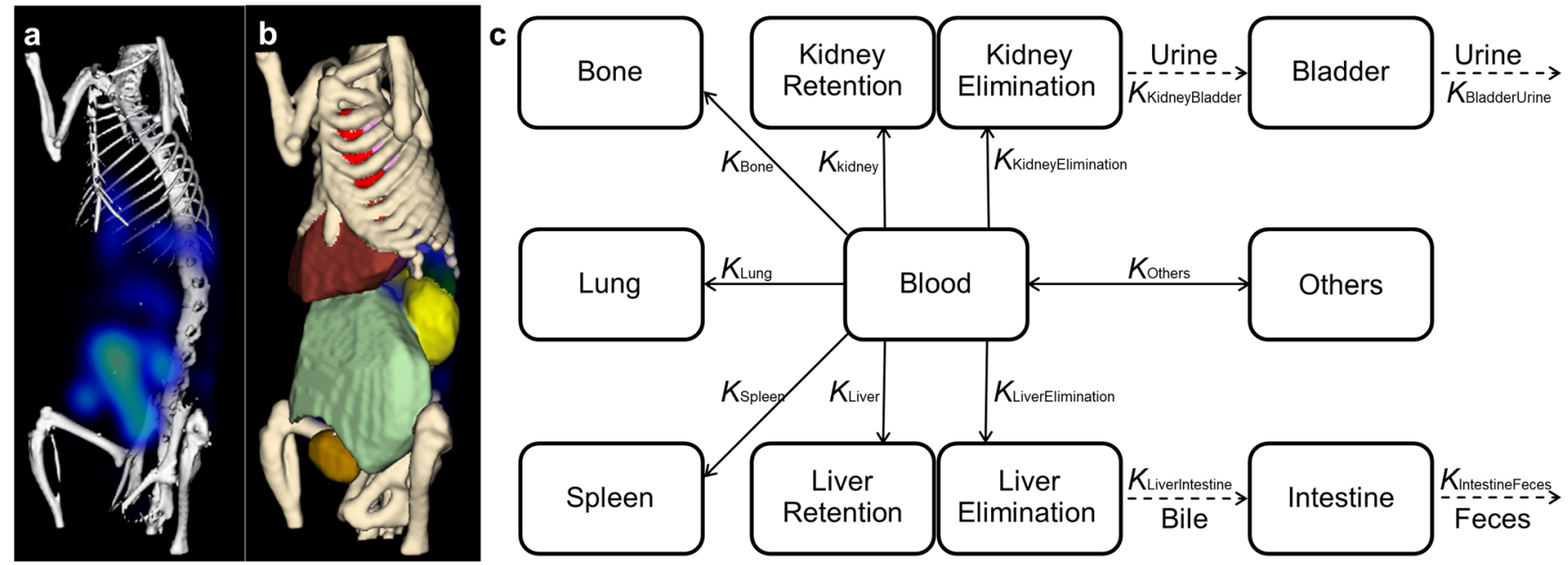

Fig. 1. Whole-body pharmacokinetic modeling using $\mu \mathrm{CT}-\mathrm{FMT}$. a Fluorescence reconstruction with $\mu \mathrm{CT}$ information, b combined with $\mu \mathrm{CT}$-based organ segmentation (heart, red; kidney, yellow; bladder, gold; liver, dark red; intestine, light green; bone, beige; spleen, green; lung, pink). c Simplified whole-body pharmacokinetic model. The blood flow (blood) facilitates compound distribution to major organs (liver, kidneys, spleen, bone, and lung). Compounds could retain in major organs and are usually eliminated by the liver or kidneys. Corresponding kinetic rates (retention rates and elimination rates) are represented by kinetic parameters $(\boldsymbol{K})$. Eight unknown kinetic parameters (solid arrows) are iteratively estimated by pharmacokinetic modeling. Four known parameters (dashed arrows) were derived from animal experiment data (from Al Rawashdeh and Zuo et al. [18]).

blood supply in some major organs, such as the liver and kidneys [6]. Based on the fused $\mu \mathrm{CT}$ and 3D fluorescence image data, the fluorescence biodistribution data (from 7 time points) of 8 organs (the heart, kidneys, bladder, liver, intestine, bone, spleen, and lung) was extracted using $\mu \mathrm{CT}$-based organ segmentation (Fig. 1b) [18].

\section{Pharmacokinetic Modeling}

Our simplified whole-body pharmacokinetic model describes the exchange of fluorescently labeled compounds between compartments [18]. This model was carefully designed to balance the number of unknown parameters and the measurements, thus achieving global convergence in simulations.

The whole-body pharmacokinetic model consists of two main elimination routes and five major retention sites. To be specific, several features are incorporated to make the model physiologically realistic and applicable in this study using intravenous injection (Fig. 1c):

(i) The blood compartment is the driving force of compound distribution (blood) [18].

(ii) Compounds are assumed to irreversibly retain in five major compartments (liver, kidney, spleen, bone, and lung) [29, 32].

(iii) Compounds are eliminated by the hepatic and/or renal elimination routes (liver, intestine, kidney, and bladder) [29].

(iv) Compounds can diffuse into the rest of the body and back into the blood (others).
Without loss of generality, the mass-balancing formulation in the context of pharmacokinetic modeling could be described as:

$\frac{d \boldsymbol{I}}{d t}=\boldsymbol{K} \boldsymbol{I}$

where vector $\boldsymbol{I}$ represents the amount of fluorescently labeled compounds in compartments, and $\boldsymbol{K}$ is the sparse adjacency matrix representing the multi-parameter set, consisting of eight unknown kinetic parameters. The unknown parameters are calculated by fitting the model to the longitudinal $\mu \mathrm{CT}$-FMT data. A constraint $0.001<$ $\boldsymbol{K}_{i j}<0.02$ is implemented in parameter estimation to ensure a physiologically meaningful range of kinetic parameters.

\section{Mixing Matrix Correction}

The fluorescence measurements in segmented organs obtained from hybrid $\mu \mathrm{CT}$-FMT are not exclusively the fluorescence intensity inside the corresponding compartments. In this study, we introduce a mixing matrix $(\boldsymbol{M})$ to "correct" the measurement of selected organs $\left(\boldsymbol{I}_{\boldsymbol{m}}\right)$ and compute the fluorescence intensity in compartments $(\boldsymbol{I})$ :

$$
\boldsymbol{I}_{\boldsymbol{m}}=\boldsymbol{M I}
$$


where $\boldsymbol{M}$ is a sum of three mixing terms:

(i) fluorescence blurring between adjacent compartments,

(ii) fluorescence blurring from regional blood vessels, and

(iii) fluorescence fusion of the elimination and retention activities of compounds inside the kidneys/liver.

The first mixing term, the fluorescence blurring from neighboring tissues due to photon diffusion and the limited spatial resolution of the FMT device, is termed "intensity diffusion" [28, 33]. To obtain the matrix of "intensity diffusion," a three-dimensional Gaussian filter (FWHM: $0.5 \mathrm{~mm}$ ) was applied to the mask of each organ segmentation. Then, the intensity average is computed in the other organ mask to obtain the corresponding "intensity diffusion" value (IDV). The IDV matrix was obtained using the hybrid $\mu$ CT-FMT data:

$\mathrm{IDV}=\left[\begin{array}{lllllllll}0.717 & 0.022 & 0.023 & 0.122 & 0.000 & 0.000 & 0.000 & 0.000 & 0.115 \\ 0.039 & 0.818 & 0.013 & 0.043 & 0.000 & 0.000 & 0.000 & 0.000 & 0.086 \\ 0.007 & 0.002 & 0.872 & 0.022 & 0.000 & 0.013 & 0.002 & 0.000 & 0.071 \\ 0.019 & 0.004 & 0.012 & 0.682 & 0.001 & 0.004 & 0.000 & 0.000 & 0.248 \\ 0.000 & 0.000 & 0.002 & 0.044 & 0.650 & 0.000 & 0.044 & 0.000 & 0.252 \\ 0.000 & 0.000 & 0.008 & 0.004 & 0.000 & 0.898 & 0.007 & 0.000 & 0.067 \\ 0.000 & 0.000 & 0.009 & 0.002 & 0.005 & 0.046 & 0.806 & 0.000 & 0.129 \\ 0.000 & 0.000 & 0.000 & 0.005 & 0.000 & 0.001 & 0.000 & 0.742 & 0.249 \\ 0.005 & 0.002 & 0.011 & 0.074 & 0.001 & 0.018 & 0.005 & 0.002 & 0.785\end{array}\right]$,

where each IDV represents the fluorescence contribution from adjacent compartments (row: lung, blood, liver, bone, spleen, intestine, kidneys, bladder, and others) to the measured organs (column: lung, blood, liver, bone, spleen, intestine, kidneys, bladder, and others).

The second mixing term represents the fluorescence blurring from vasculature inside the organs, determined by the relative blood volume of organs (rBV). The rBV data were obtained from previous studies [34].

The third mixing term incorporates the elimination and retention sub-compartments in the liver and kidneys (Fig. 1c). The mixing matrix involving these three mixing terms was included in the forward model and the iterative model fitting to deconvolute the kinetic parameters.

\section{Kinetic Parameter Estimation}

With the mixing matrix-corrected forward model, an iterative strategy based on the kinetic model was applied to estimate the best-fit kinetic parameters.

\section{(i) Mixing Matrix-Corrected Kinetic Modeling}

A fine-grained prediction $\widehat{\boldsymbol{I}}_{\boldsymbol{f}}$ (Fig. 2a) is computed by applying the 4th-order Runge-Kutta algorithm to the differential equation (Eq. 1): $\frac{d \widehat{\boldsymbol{I}}_{\boldsymbol{f}}}{d t}=\boldsymbol{K} \widehat{\boldsymbol{I}}_{\boldsymbol{f}}$.

This prediction is computed at a sufficiently high temporal resolution to avoid numerical errors stemming from using coarse steps in the Runge-Kutta method. The fine prediction $\widehat{\boldsymbol{I}}_{\boldsymbol{f}}$ is then coarsely sampled to obtain the coarse prediction $\widehat{\boldsymbol{I}}_{\boldsymbol{c}}$ (Fig. 2b) at defined time points, such as the 7 time points at $0.25,2,4,8,24,48$, and $72 \mathrm{~h}$ in the in vivo experiments. $\widehat{\boldsymbol{I}}_{\boldsymbol{c}}$ is further modified by the mixing matrix $\boldsymbol{M}$ to generate the mixed prediction $\widehat{\boldsymbol{I}}_{\boldsymbol{m}}$-the prediction of measurement in organs (Fig. 2c):

$\widehat{\boldsymbol{I}}_{\boldsymbol{m}}=\boldsymbol{M} \widehat{\boldsymbol{I}}_{\boldsymbol{c}}$

\section{(ii) Cost Function-Based Parameter Estimation}

To estimate the best-fit $\boldsymbol{K}$, the cost function is defined as:

$\boldsymbol{f}(\boldsymbol{K})=\sum\left(\widehat{\boldsymbol{I}}_{\boldsymbol{m}}-\boldsymbol{I}_{\boldsymbol{m}}\right)^{2}+\boldsymbol{c} \boldsymbol{K}^{2}$

In this model fitting problem, the first part $\sum\left(\widehat{\boldsymbol{I}}_{\boldsymbol{m}}-\boldsymbol{I}_{\boldsymbol{m}}\right)^{2}$ represents the sum-squared error between the prediction $\widehat{\boldsymbol{I}}_{\boldsymbol{m}}$ (Fig. 2c) and measurement $\boldsymbol{I}_{\boldsymbol{m}}$ (Fig. 2d). The regularization term $\boldsymbol{c} \boldsymbol{K}^{2}$ is a parameter penalty to avoid extremely large solutions of $\boldsymbol{K}$.

Then, the nonlinear conjugate gradient method is used to minimize the penalized cost function $\boldsymbol{f}(\boldsymbol{K})$. Iteratively, gradients are computed using adjoint algorithmic differentiation to determine a descent direction and perform a line search [35]. Accordingly, the eight unknown kinetic parameters are estimated by iteratively conducting these two steps.

\section{Simulations}

Numerical simulations were performed to assess whether kinetic parameters can be deconvoluted using this model and to estimate the effect of the mixing matrix. One hundred groups of kinetic parameters were randomly generated, independently and uniformly distributed in the range 0.001 to 0.02 . Fine measurements $\widehat{\boldsymbol{I}}_{\boldsymbol{f}}$ were then simulated based on the proposed kinetic modeling. Coarsely sampled measurements $\widehat{\boldsymbol{I}}_{\boldsymbol{c}}$ were extracted from the fine measurements $\widehat{\boldsymbol{I}}_{\boldsymbol{f}}$ at $0.25,2,4,8,24,48$, and $72 \mathrm{~h}$. Besides, a $10 \%$ relative noise was randomly added to the sampled measurements. With the noisy measurements, the mixing matrix-corrected model was applied to estimate the kinetic parameters. 

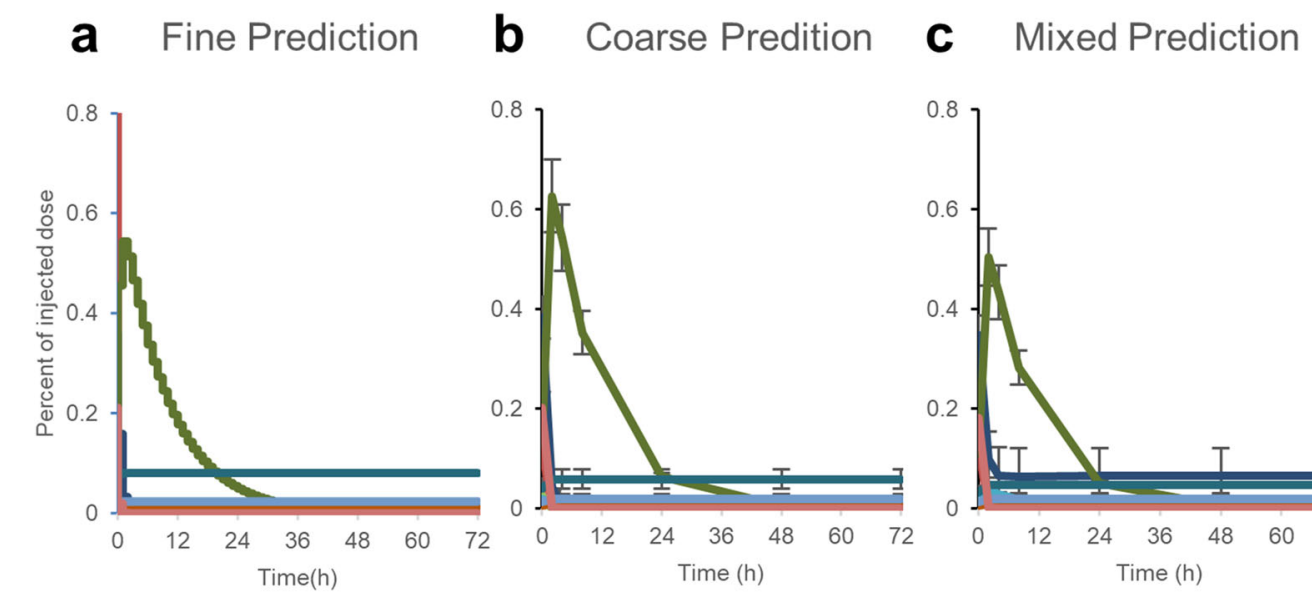

\section{d Measurement}
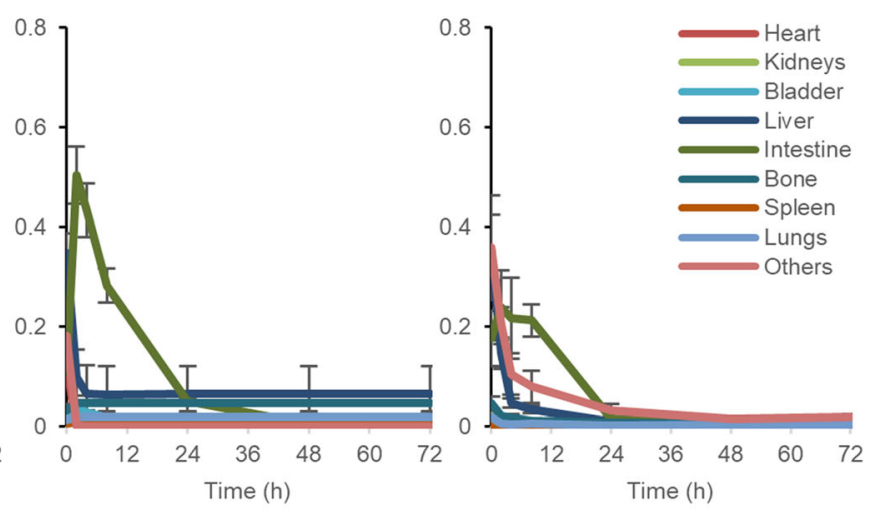

Fig. 2. Modeling steps of the pharmacokinetic curves. a Fine-grained (high temporal resolution) prediction $\widehat{\boldsymbol{I}}_{\boldsymbol{f}}$ is generated based on the pharmacokinetic model. b Coarse prediction $\widehat{\boldsymbol{I}}_{\boldsymbol{c}}$ is then extracted from the fine curves at sparse time points $0.25,2,4,8,24,48$, and $72 \mathrm{~h}$ after i.v. injection. c Mixed prediction $\widehat{\boldsymbol{I}}_{\boldsymbol{m}}$ is calculated by multiplying the coarse prediction $\widehat{\boldsymbol{I}}_{\boldsymbol{c}}$ using the mixing matrix. $\mathbf{d}$ The measurement $\boldsymbol{I}_{\boldsymbol{m}}$ is from animal experiment data using hybrid $\mu$ CT-FMT (ICG) [18].

The performance of the kinetic parameter estimation with mixing matrix correction was studied and compared to the estimation without correction. The feasibility and reliability of the corrected modeling were evaluated in terms of the linear relationships of parameters and the relative errors of the estimated parameters compared to the uncorrected modeling.

To investigate the dependency of the corrected modeling on sampling density, different temporal sampling schemes were applied. More densely sampled measurements $\widehat{\boldsymbol{I}}_{\boldsymbol{c}}$ (at 7, 20, and 50 time points) were extracted from the fine measurements $\widehat{\boldsymbol{I}}_{\boldsymbol{f}}$. Furthermore, the corrected modeling with more early sampling points at the pharmacokinetic process, such as 7-early, i.e., seven measurements at $0,0.25,0.5,2,12,24$, and $48 \mathrm{~h}$, more densely sampled in the first hour, was investigated to explore the influence of data sampling during the early phase of probe distribution.

\section{Statistical Analysis}

In simulations, linear regression analysis was used to examine the linear relationship and the goodness of fit between the randomly chosen and estimated parameters. In the in vivo study, the kinetic parameters estimated by the uncorrected and mixing matrix-corrected modeling were compared using a paired two-tailed t-test.

\section{Results}

\section{Simulations}

Mathematical simulations were designed to evaluate if the mixing matrix-corrected model could recover the kinetic parameters and to assess the effect of the mixing matrix on the parameter estimation.

Figure 3 shows the comparison of kinetic parameters estimated by the corrected and uncorrected modeling. With mixing matrix correction, all eight estimated parameters are approximately linear related to simulated parameters (slopes close to 1 and high R-squared values). In contrast, the parameters estimated by the uncorrected model have higher variances and are less linear, especially the hepatic and renal retention parameters. For example, the slope of the hepatic retention rate $\left(K_{\text {Liver }}\right)$ is $1.042 \pm 0.014$ with correction, whereas the uncorrected modeling is $-0.009 \pm 0.018$ (Fig. 4). The strong linear relationship between the estimated and simulated parameters confirmed that the mixing matrix correction could significantly improve the parameter estimation in whole-body pharmacokinetic modeling.

The relative errors of kinetic parameters were also compared. Figure 4 shows the error distribution of the estimated parameters using the corrected and uncorrected modeling. With mixing matrix correction, estimated parameters have smaller relative errors, especially for the kidney and liver's elimination and retention rates. Despite $10 \%$ noise added to the measurements, the kinetic parameters estimated by the corrected modeling only have an average relative error of $9.77 \%$, compared to the average relative error of $98.41 \%$ by the uncorrected modeling. Additionally, we found that larger simulated parameters $(>0.005)$ could result in smaller relative errors in parameter estimation, which is reasonable because small parameter changes are hard to estimate under the presence of noise.

To assess the dependency of parameter estimation on sampling density, we investigated the effect of different temporal sampling schemes on parameter estimation using mixing matrix-corrected modeling.

Table 1 shows that sampling with more data points reduces the relative errors of estimated parameters. Moreover, the same amounts of measurements with more early samples in the pharmacokinetics (especially within the first hour) can suppress much more noise in parameter estimation, such as the "7-early" sampling scheme with $5.76 \%$ 

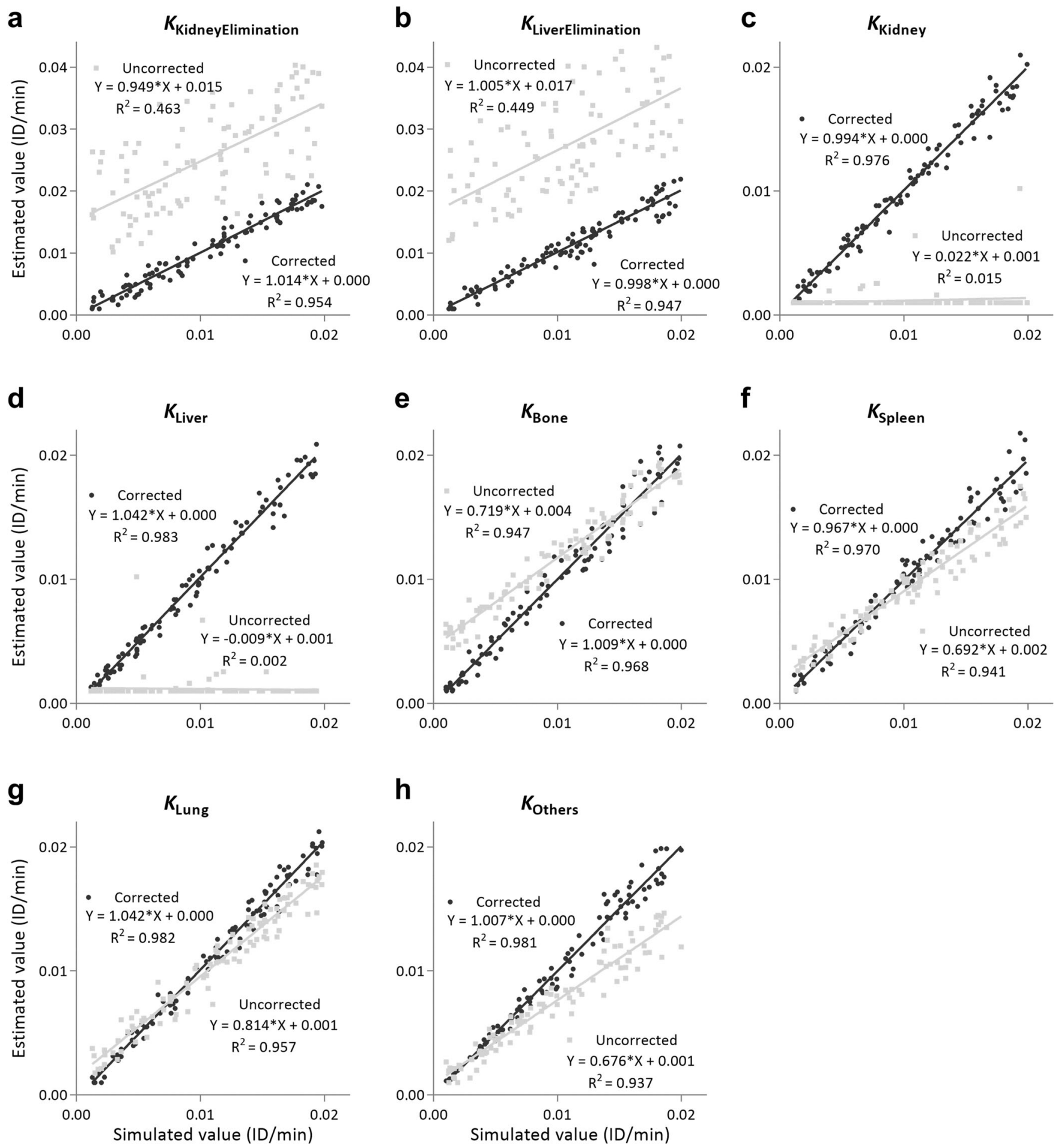

Fig. 3. Comparison of estimated parameters using the uncorrected and mixing matrix-corrected kinetic modeling in simulations. Measurements were simulated using the mixing matrix, and random noise was added. Only the estimates using the mixing matrix have strong linear relationships with the original parameters (slopes and R-squared close to 1) for all parameters. a, b Without the mixing matrix, the kidney and liver's elimination rates, the estimates have higher variances (small R-squared values) and a higher values bias. c, d Without the mixing matrix, the renal and hepatic retention rates cannot be recovered. $\mathbf{e}-\mathbf{h}$ The other estimated parameters (bone, spleen, lung, and others) with uncorrected modeling have less linear fit lines.

averaged relative errors compared to the "7" sampling scheme with $9.77 \%$. The noise reduction is reasonable because of the fast exchange of compounds between compartments in the early phase of probe distribution. 

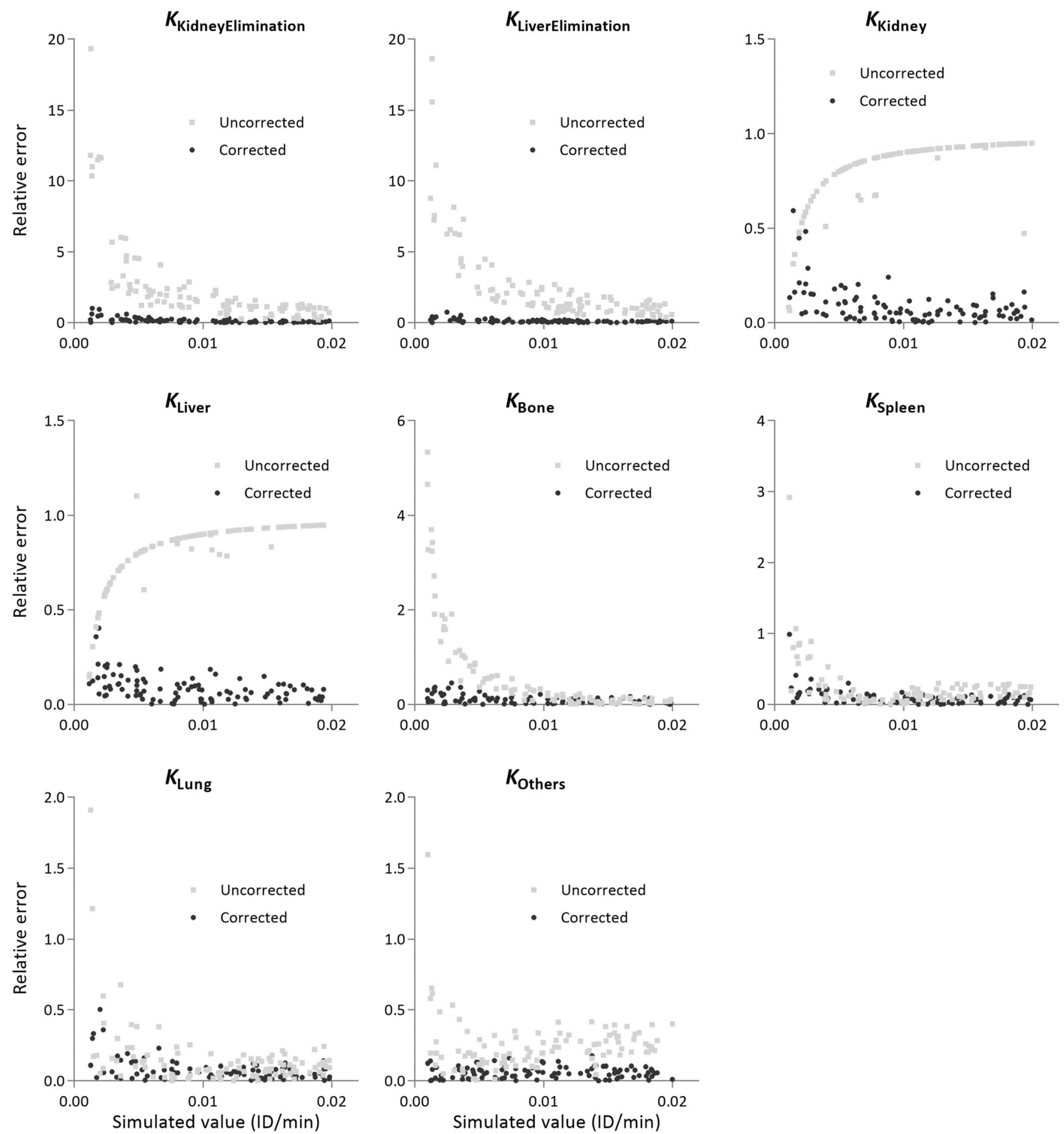

Fig. 4. Relative errors of estimated parameters using the uncorrected and mixing matrix-corrected kinetic modeling in simulations. Measurements were simulated using the mixing matrix, and random noise was added. The relative errors of estimated parameters are notably smaller using mixing matrix correction, especially for the kidney and liver's elimination and retention rates. Smaller relative errors are achieved with parameters larger than 0.005 . Simulated parameters are randomly generated, to obtain simulated measurements.

\section{In Vivo Study}

The kinetic parameters of four fluorescently labeled compounds were estimated using the mixing matrix-corrected modeling and the uncorrected one using longitudinal $\mu \mathrm{CT}$ FMT imaging data. The kinetic modeling consists of seven kinetic parameters consisting of two elimination routes and five retention sites. 
Table 1. Relative errors of kinetic parameters with six temporal sampling schemes using the mixing matrix-corrected modeling. 7-early means 7 measurements with more dense sampling in the early pharmacokinetics (especially within the first hour), 20 -early and 50 -early in a similar way. A $10 \%$ relative noise is randomly added to the simulated measurements

\begin{tabular}{|c|c|c|c|c|c|c|}
\hline \multirow[b]{2}{*}{ Temporal sampling } & \multicolumn{6}{|c|}{ Relative error } \\
\hline & 7 & 20 & 50 & 7-early & 20-early & 50-early \\
\hline$K_{\text {KidneyElimination }}$ & $14.9 \%$ & $13.9 \%$ & $12.5 \%$ & $10.3 \%$ & $6.37 \%$ & $4.88 \%$ \\
\hline$K_{\text {LiverElimination }}$ & $12.2 \%$ & $12.8 \%$ & $11.4 \%$ & $6.60 \%$ & $4.78 \%$ & $4.62 \%$ \\
\hline$K_{\text {Kidney }}$ & $8.65 \%$ & $5.99 \%$ & $5.23 \%$ & $4.26 \%$ & $3.83 \%$ & $3.72 \%$ \\
\hline$K_{\text {Liver }}$ & $8.69 \%$ & $6.19 \%$ & $5.58 \%$ & $5.29 \%$ & $3.89 \%$ & $3.66 \%$ \\
\hline$K_{\text {Bone }}$ & $10.6 \%$ & $7.74 \%$ & $6.01 \%$ & $5.41 \%$ & $3.84 \%$ & $3.57 \%$ \\
\hline$K_{\text {Spleen }}$ & $9.49 \%$ & $7.32 \%$ & $5.79 \%$ & $4.65 \%$ & $3.97 \%$ & $3.78 \%$ \\
\hline$K_{\text {Lung }}$ & $7.50 \%$ & $6.08 \%$ & $5.05 \%$ & $4.40 \%$ & $3.82 \%$ & $3.58 \%$ \\
\hline$K_{\text {Others }}$ & $6.14 \%$ & $6.44 \%$ & $6.48 \%$ & $5.17 \%$ & $5.29 \%$ & $5.14 \%$ \\
\hline Average relative error & $9.77 \%$ & $8.31 \%$ & $7.26 \%$ & $5.76 \%$ & $4.47 \%$ & $4.12 \%$ \\
\hline
\end{tabular}

\section{Elimination}

Figure 5 shows that ICG, MB, and $\mathrm{NG}$ are mainly eliminated by the liver, whereas OS is eliminated by the liver and kidneys. It was noted that using the mixing matrix correction, the hepatic elimination rate of ICG and the renal elimination rate of OS were slightly larger, compared to the rates without the correction (OS: $\mathrm{P}<0.05)$ (Fig. 5a, d). However, the hepatic elimination rates of $\mathrm{MB}$ and $\mathrm{NG}$ were significantly smaller using the mixing matrix $(\mathrm{P}<0.01$ and $\mathrm{P}<0.01$, respectively) (Fig. 5b, c).

\section{Retention}

Figure 6 shows that the usage of the mixing matrix correction lowers the variances of retention parameters. $\mathrm{MB}$ and NG were mainly retained in the liver (Fig. 6b, c). With correction, the renal retention rates of ICG, $\mathrm{MB}, \mathrm{NG}$, and $\mathrm{OS}$ were smaller (MB: $\mathrm{P}<0.05$ and OS: $\mathrm{P}<0.01$, respectively). With the corrected modeling, OS had a significantly higher retention rate in the bone $(2.06 \pm 0.37 \% \mathrm{ID} / \mathrm{min})$ than in the kidneys $(0.10 \pm 0.00 \% \mathrm{ID} /$ $\mathrm{min})$ and liver $(0.10 \pm 0.00 \% \mathrm{ID} / \mathrm{min})$, consistent with the bonespecific property of OS. In addition, the corrected bone retention rate of OS $(2.06 \pm 0.37 \% \mathrm{ID} / \mathrm{min})$ was significantly larger, compared to ICG, MB, and NG $(0.95 \pm 0.60,1.17 \pm 0.46$, and $0.62 \pm 0.26 \% \mathrm{ID} / \mathrm{min}$ respectively). In the corrected modeling, slightly larger spleen and lung retention rates were obtained for all four probes.

The corrected modeling incorporated the retention and elimination sub-compartments in the liver. This incorporation resulted in slightly lower hepatic elimination rates of MB (Fig. 5b, $\mathrm{P}<0.01$ ) and NG (Fig. 5c, $\mathrm{P}<0.01$ ) but higher hepatic retention rates of MB (Fig. 6b, $\mathrm{P}<0.05$ ) and NG (Fig. $6 \mathrm{c}, \mathrm{P}<0.05$ ). Additionally, the renal and hepatic retention rates of OS were significantly smaller using correction (Fig. $6 \mathrm{~d}, \mathrm{P}<0.01$ and $\mathrm{P}<0.01$, respectively).

\section{Discussion}

Pharmacokinetic modeling is a useful tool to obtain kinetic parameters and facilitates a better understanding of compounds' kinetic behaviors. Our study aimed to improve the parameter estimation using mixing matrix correction based on longitudinal $\mu \mathrm{CT}$-FMT data. The mixing matrix "corrected" the measured pharmacokinetic curves, and actual fluorescence intensities of compartments were thus computed. The correction of fluorescence measurements could avoid serious bias in parameter estimation in this study using intravenous injection, especially for compounds distinctively retained in the liver and/or kidneys.

In the simulations, when using the mixing matrixcorrected modeling, stronger linear relationships between the estimated and simulated parameters were achieved, compared to the uncorrected one. The linear relationships of the renal and hepatic retention rates mainly depend on the differentiation of the retention and elimination subcompartments in the liver and kidneys. Smaller relative errors of estimated parameters are expected since the mixing matrix-corrected modeling minimizes the influence of fluorescence blurring from surrounding organs and the regional blood flow in well-perfused organs, especially in the liver, kidneys, and bone. The high degree of accuracy in parameter estimation indicates that the proposed modeling with mixing matrix correction is robust and reliable.

We also found that sampling with more data points, mostly significantly sufficient measurements in the early pharmacokinetics (particularly within the first hour), could notably suppress the noise and reduce relative errors in parameter estimation. However, the time sampling scheme in the animal experiment is highly limited by the performance of the hybrid $\mu$ CT-FMT imaging device (13 min per hybrid $\mu$ CT-FMT scan) $[17,18]$. Although the inclusion of $\mu \mathrm{CT}$ imaging provides a more accurate localization of fluorescently labeled compounds [6, 18], it extends the scanning duration and limits the amount of sampling in the early phase. Important pharmacokinetic information is lost because of insufficient measurements from the early stage of probe distribution. The limitation could be overcome by including temporal averaging in the model, or by using a faster device [36]. Such a faster FMT could be developed using CMOS cameras. These relatively inexpensive and fast cameras are smaller, and multiple cameras could be used, 

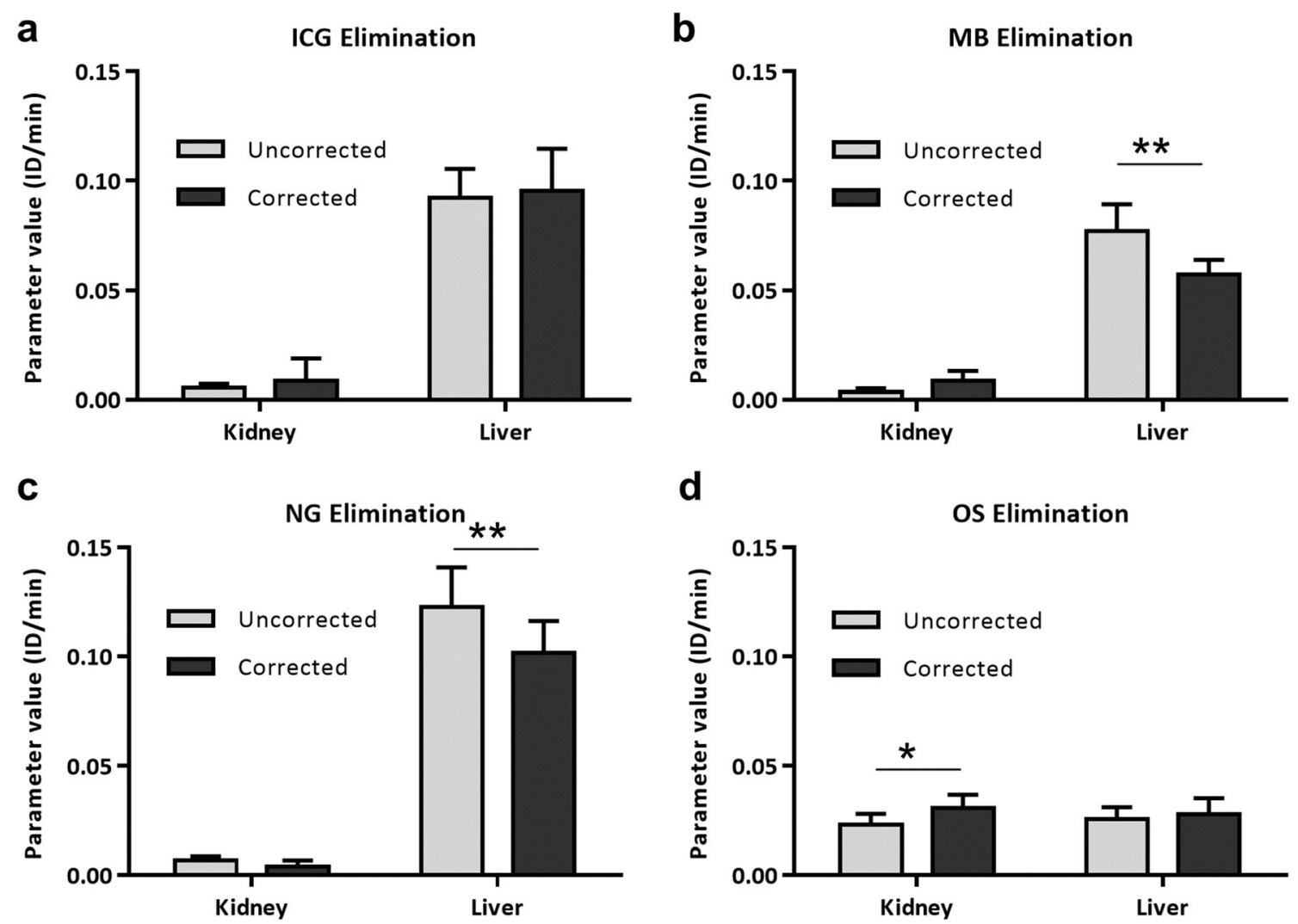

Fig. 5. Hepatic and renal elimination of ICG, MB, NG, and OS using the uncorrected and mixing matrix-corrected modeling. ac ICG, MB, and NG are mainly eliminated by the liver. d With correction, the renal elimination rate of OS was slightly higher compared to the rates without the correction $(\mathrm{P}<0.05)$. b, $\mathbf{c} \mathrm{MB}$ and NG's hepatic elimination rates are smaller using correction $(P<0.01$ and $P<0.01$, respectively). The paired two-tailed t-test was used in the comparison of elimination rates between the corrected and uncorrected modeling using $\mu$ CT-FMT data, $n=5$.

e.g., one for the excitation and one for the emission channel. Multiple lasers could be used and operated in interleaved mode, requiring less physical movement.

In the in vivo results, the estimated parameters are physiologically meaningful, consistent with the expected elimination and retention properties of these four compounds [18]. ICG and NG had the highest hepatic elimination rates, while OS had the highest renal elimination rate. The hepatic retention was highest for $\mathrm{MB}$ and $\mathrm{NG}$, while bone retention was highest for OS.

For the assessment of compound elimination in the in vivo data analysis, a minor but noteworthy change is that, for OS elimination, the renal elimination rate is slightly larger than the hepatic elimination rate using the mixing matrix-corrected modeling. This is physiologically meaningful since probes with a hydrodynamic size below the glomerular filtration threshold $(<8 \mathrm{~nm})$ and positively charged typically undergo substantial renal clearance [29, 31].

The retention rates of compounds were significantly different when using the mixing matrix correction. The hepatic retention rates of $\mathrm{MB}$ and $\mathrm{NG}$ were considerably larger than the renal retention rates, which is consistent with previous studies [29, 30]. The prolonged retention of these compounds is caused by the rapid uptake but relatively slow excretion by the liver. The bone retention rate of OS was significantly larger than ICG, MB, and NG, consistent with OS's bone-specific property. Additionally, the spleen and lung retention rates of $\mathrm{ICG}, \mathrm{MB}$, and $\mathrm{NG}$ are slightly larger. This is reasonable because of the uptake by the mononuclear phagocyte system in these organs [29, 31, 37, 38]. However, the overestimation of the lung retention rate for OS might be a result of the fluorescence spillover in the lung from the neighboring bone tissues [18, 39].

Besides, the pharmacokinetic properties of the fluorescently labeled compounds, i.e., $\mathrm{MB}$ and $\mathrm{NG}$, might be influenced by numerous aspects, such as the charge, size, shape surface modifications, solubility, and affinity to plasma proteins or specific receptors [18]. The proposed kinetic modeling can be used to investigate the impact of fluorescence labeling on pharmacokinetic properties, e.g., by comparing compounds with different fluorescent labeling methods.

In this study, some modifications were adopted in the pharmacokinetic modeling, based on the previously presented compartmental modeling [25, 40]. The proposed 

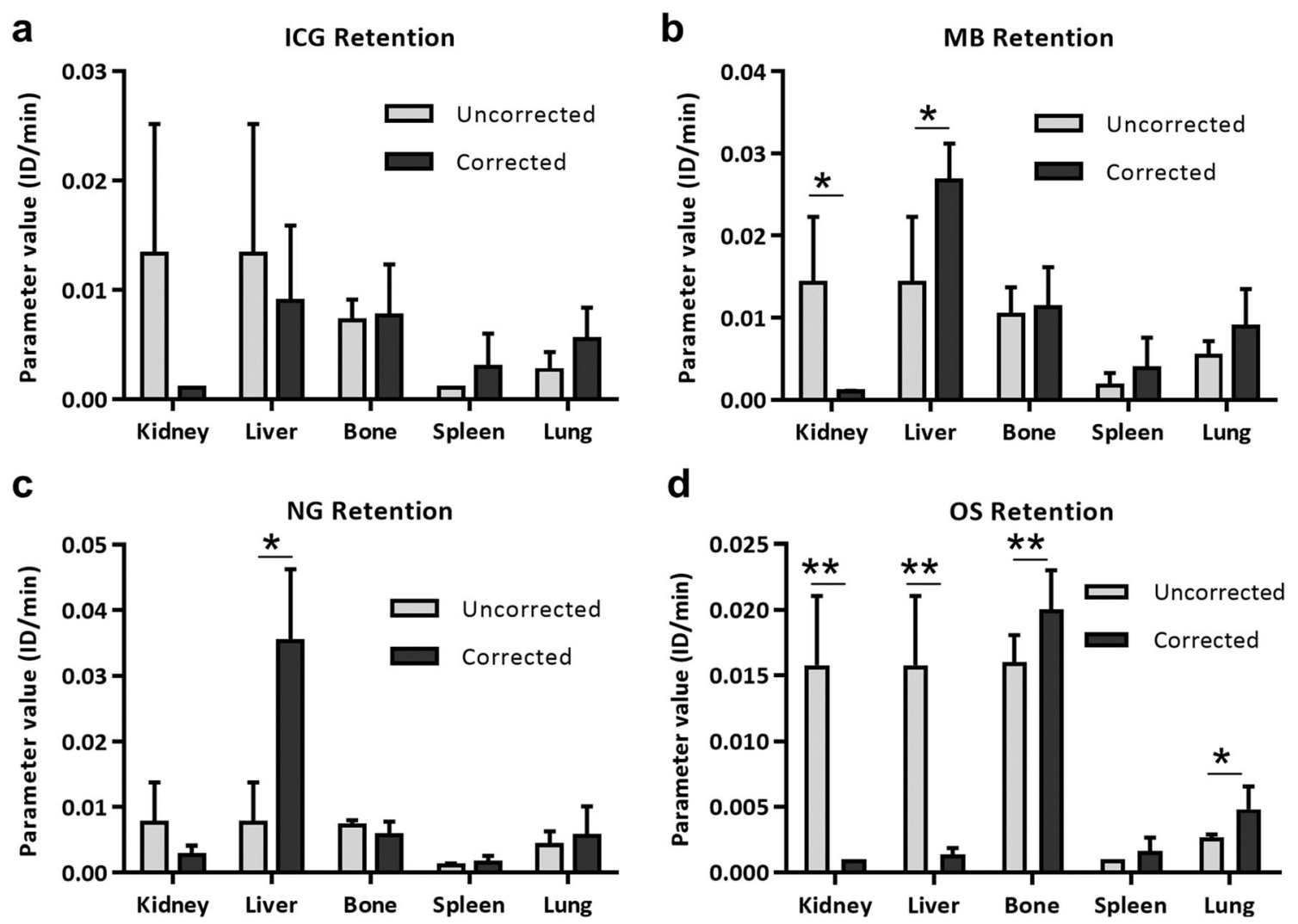

Fig. 6. Retention of ICG, MB, NG, and OS in selected organs using the uncorrected and mixing matrix-corrected modeling. ad ICG, MB, NG, and OS had negligible renal retention rates when using the corrected modeling compared to the uncorrected one. b, $\mathbf{c} M B$ and NG show a higher hepatic retention rate $(P<0.05$ and $P<0.05$, respectively). $\mathbf{d}$ With correction, OS has a higher bone retention rate $(P<0.01)$, while lower renal and hepatic retention rates $(P<0.01$ and $P<0.01$, respectively). The paired twotailed t-test was used in the comparison of elimination rates between the corrected and uncorrected modeling using $\mu \mathrm{CT}$-FMT data, $\mathrm{n}=5$.

whole-body modeling incorporates the main elimination routes and major retention sites, which comprise the complete biodistribution of compounds inside the body. The bone targeting property of OS was observed in the animal results, compared to ICG, MB, and NG. This suggests that our proposed modeling can be applied to other fluorescent compounds targeting specific cells and tissues, e.g., a tumor-targeting fluorescent probe.

In this model, the retention sites are simplified only as trapped compartments, where the transfer of compounds between compartments is irreversible. The irreversible retention is physiologically meaningful and suitable, representing the trap of macromolecules in the liver, such as albumin-binding ICG, MB, and NG [29, 31, 37, 38]. However, in some whole-body modeling studies, especially in PET using small radiolabeled compounds with reversible binding to specific organs, the transfer between the blood and tissue compartments is reversible [41, 42]. Besides, limited by the image quality and the temporal resolution, a good blood signal curve was not available in the $\mu$ CT-FMT kinetic modeling, especially in the early phase of the pharmacokinetic process. In contrast, with high temporal resolution $(\sim$ seconds), PET kinetic modeling strongly depends on the "arterial input function" and good image quality. This transfer property and the "arterial input function" should be reconsidered to extrapolate the model to PET kinetic data in future studies. Furthermore, considering the complexity of the hepatobiliary elimination and the limited resolution of FMT, the liver is regarded as an isolated organ, whereas $80 \%$ of the hepatic blood supply is from the spleen, gut, and pancreas [43, 44]. These simplifications of the hepatic elimination route need to be reconsidered in animal studies, especially using oral administration.

In the hybrid $\mu$ CT-FMT imaging, anesthetized mice were imaged to obtain pharmacokinetic curves. Motion artifacts caused by breathing and cardiac movements contribute to fluorescence blurriness and result in inaccurate biodistribution data. Therefore, a mouse bed was applied in the experiments to restrain mice's motion and limit the impact on fluorescence measurement [17], though, in future in vivo studies, the remaining motion artifact problems could be considered to improve the pharmacokinetic analysis.

Despite these limitations, we have demonstrated that the proposed whole-body modeling can improve the parameter estimation with a significantly higher degree of accuracy by 
involving a mixing matrix. With the new approach, the differences in retention sites can be shown notably more explicit using $\mu$ CT-FMT animal data. This indicates that the corrected modeling might be a promising tool to assess the pharmacokinetics of organ-specific compounds using in vivo data.

Author Contribution. S. Z. proposed the kinetic modeling method, validated the modeling in simulation analysis and using in vivo data, and wrote the original manuscript. WA. R. conducted the animal experiments, and collected, and analyze the in vivo data. S. R., Z. M., and Y. G. reviewed the article. F. G. and F. K.supervised the study and reviewed the article.

Funding. Open Access funding enabled and organized by Projekt DEAL. The authors would like to thank the Chinese Scholarship Council, the Federal Government of North-Rhine Westphalia, and the European Regional Development Fund, the START-Program of the RWTH Aachen Faculty of Medicine, and the German Research Foundation (GR 5027/2-1 and CRC1382 project ID 403224013 - SFB 1382, project Q1) for funding. Performance measurements were performed with computing resources granted by RWTH Aachen University under project rwth0178.

\section{Declarations}

\section{Conflict of Interest}

Fabian Kiessling is section editor of Molecular Imaging and Biology.

\section{Ethics Statement}

All animal experimental procedures were approved by the Governmental Review Committee on Animal Care.

\section{Disclosure}

Felix Gremse is the owner of Gremse-IT GmbH, a spin-out of the RWTH Aachen University, which commercializes software for biomedical image analysis.

Open Access This article is licensed under a Creative Commons Attribution 4.0 International License, which permits use, sharing, adaptation, distribution and reproduction in any medium or format, as long as you give appropriate credit to the original author(s) and the source, provide a link to the Creative Commons licence, and indicate if changes were made. The images or other third party material in this article are included in the article's Creative Commons licence, unless indicated otherwise in a credit line to the material. If material is not included in the article's Creative Commons licence and your intended use is not permitted by statutory regulation or exceeds the permitted use, you will need to obtain permission directly from the copyright holder. To view a copy of this licence, visit http:// creativecommons.org/licenses/by/4.0/.

\section{References}

1. Ntziachristos V, Tung CH, Bremer C, Weissleder R (2002) Fluorescence molecular tomography resolves protease activity in vivo. Nat Med 8:757-761

2. Ntziachristos V, Ripoll J, Wang LV, Weissleder R (2005) Looking and listening to light: the evolution of whole-body photonic imaging. Nat Biotechnol 23:313-320

3. Patwardhan SV, Bloch SR, Achilefu S, Culver JP (2005) Timedependent whole-body fluorescence tomography of probe biodistributions in mice. Opt Express 13:2564-2577

4. Deliolanis N, Lasser T, Hyde D, Soubret A, Ripoll J, Ntziachristos V (2007) Free-space fluorescence molecular tomography utilizing 360 geometry projections. Opt Lett 32:382-384

5. Deliolanis NC, Ale A, Morscher S, Burton NC, Schaefer K, Radrich K, Razansky D, Ntziachristos V (2014) Deep-tissue reporter-gene imaging with fluorescence and optoacoustic tomography: a performance overview. Mol Imaging Biol 16:652-660

6. Gremse F, Theek B, Kunjachan S, Lederle W, Pardo A, Barth S, Lammers T, Naumann U, Kiessling F (2014) Absorption reconstruction improves biodistribution assessment of fluorescent nanoprobes using hybrid fluorescence-mediated tomography. Theranostics 4:960 971

7. Rosenhain S, Al Rawashdeh W, Kiessling F, Gremse F (2017) Sensitivity and accuracy of hybrid fluorescence-mediated tomography in deep tissue regions. J Biophotonics 10:1208-1216

8. Vasquez KO, Casavant C, Peterson JD (2011) Quantitative whole body biodistribution of fluorescent-labeled agents by non-invasive tomographic imaging. PLoS One 6:e20594

9. Peterson NC, Wilson GG, Huang Q, Dimasi N, Sachsenmeier KF (2016) Biodistribution analyses of a near-infrared, fluorescently labeled, bispecific monoclonal antibody using optical imaging. Comp Med 66:90-99

10. Heller DA, Levi Y, Pelet JM, Doloff JC, Wallas J, Pratt GW, Jiang S, Sahay G, Schroeder A, Schroeder JE, Chyan Y, Zurenko C, Querbes W, Manzano M, Kohane DS, Langer R, Anderson DG (2013) Modular 'click-in-emulsion' bone-targeted nanogels. Adv Mater 25:1449-1454

11. Zaheer A, Lenkinski RE, Mahmood A, Jones AG, Cantley LC, Frangioni JV (2001) In vivo near-infrared fluorescence imaging of osteoblastic activity. Nat Biotechnol 19:1148-1154

12. Ale A, Ermolayev V, Herzog E, Cohrs C, de Angelis MH, Ntziachristos V (2012) FMT-XCT: in vivo animal studies with hybrid fluorescence molecular tomography-X-ray computed tomography. Nat Methods 9:615-620

13. Kunjachan S, Gremse F, Theek B, Koczera P, Pola R, Pechar M, Etrych T, Ulbrich K, Storm G, Kiessling F, Lammers T (2013) Noninvasive optical imaging of nanomedicine biodistribution. ACS Nano $7: 252-262$

14. Hyde D, Dekleine R, Maclaurin S et al (2009) Hybrid FMT-CT imaging of amyloid- $\beta$ plaques in a murine Alzheimer's disease model. NeuroImage 44:1304-1311

15. Ntziachristos V (2010) Going deeper than microscopy: the optical imaging frontier in biology. Nat Methods 7:603-614

16. Yalavarthy PK, Pogue BW, Dehghani H, Paulsen KD (2007) Weightmatrix structured regularization provides optimal generalized leastsquares estimate in diffuse optical tomography. Med Phys 34:20852098

17. Gremse F, Doleschel D, Zafarnia S et al (2015) Hybrid $\mu$ CT-FMT imaging and image analysis. J Vis Exp 100:e52770

18. Al Rawashdeh W, Zuo S, Melle A et al (2017) Noninvasive assessment of elimination and retention using CT-FMT and kinetic whole-body modeling. Theranostics 7:1499-1510

19. Gremse F, Stärk M, Ehling J, Menzel JR, Lammers T, Kiessling F (2016) Imalytics preclinical: interactive analysis of biomedical volume data. Theranostics 6:328-341

20. Dai Y, Yin J, Huang Y, Chen X, Wang G, Liu Y, Zhang X, Nie Y, Wu K, Liang J (2016) In vivo quantifying molecular specificity of Cy55-labeled cyclic 9-mer peptide probe with dynamic fluorescence imaging. Biomed Opt Express 7:1149-1159

21. Brix G, Griebel J, Kiessling F, Wenz F (2010) Tracer kinetic modelling of tumour angiogenesis based on dynamic contrastenhanced CT and MRI measurements. Eur J Nucl Med Mol Imaging 37:30-51

22. Guang H, Cai C, Zuo S, Cai W, Zhang J, Luo J (2017) Multiparametric evaluation of hindlimb ischemia using time-series indocyanine green fluorescence imaging. J Biophotonics 10:456-464

23. Elliott JT, Samkoe KS, Davis SC, Gunn JR, Paulsen KD, Roberts DW, Pogue BW (2016) Image-derived arterial input function for quantitative fluorescence imaging of receptor-drug binding in vivo. $\mathrm{J}$ Biophotonics 9:282-295

24. Jiang D, Im H-J, Boleyn ME, England CG, Ni D, Kang L, Engle JW, Huang P, Lan X, Cai W (2019) Efficient renal clearance of DNA tetrahedron nanoparticles enables quantitative evaluation of kidney function. Nano Res 12:637-642

25. Huang J, O'Sullivan F (2014) An analysis of whole body tracer kinetics in dynamic pet studies with application to image-based blood input function extraction. IEEE Trans Med Imaging 33:1093-1108

26. Grudzinski JJ, Floberg JM, Mudd SR, Jeffery JJ, Peterson ET, Nomura A, Burnette RR, Tomé WA, Weichert JP, Jeraj R (2012) 
Application of a whole-body pharmacokinetic model for targeted radionuclide therapy to NM404 and FLT. Phys Med Biol 57:16411657

27. Yun SH, Kwok SJJ (2017) Light in diagnosis, therapy and surgery. Nat Biomed Eng 1:1-16

28. Skretting A (2009) Intensity diffusion' is a better description than 'partial volume effect. Eur J Nucl Med Mol Imaging 36:536-537

29. Longmire M, Choyke PL, Kobayashi H (2008) Clearance properties of nano-sized particles and molecules as imaging agents: considerations and caveats. Nanomed 3:703-717

30. Palmowski M, Morgenstern B, Hauff P et al (2008) Pharmacodynamics of streptavidin-coated cyanoacrylate microbubbles designed for molecular ultrasound imaging. Investig Radiol 43:162-169

31. Ergen C, Heymann F, Al Rawashdeh W et al (2017) Targeting distinct myeloid cell populations in vivo using polymers, liposomes and microbubbles. Biomaterials 114:106-120

32. Garg A, Balthasar JP (2007) Physiologically-based pharmacokinetic (PBPK) model to predict IgG tissue kinetics in wild-type and FcRnknockout mice. J Pharmacokinet Pharmacodyn 34:687-709

33. Zhang X, Gong Y, Li Y et al (2017) Image restoration for fluorescence planar imaging with diffusion model. Biomed Res Int 2017:1-18

34. Boswell CA, Mundo EE, Ulufatu S, Bumbaca D, Cahaya HS, Majidy N, van Hoy M, Schweiger MG, Fielder PJ, Prabhu S, Khawli LA (2014) Comparative physiology of mice and rats: radiometric measurement of vascular parameters in rodent tissues. Mol Pharm 11:1591-1598.

35. Gremse F, Höfter A, Razik L, Kiessling F, Naumann U (2016) GPUaccelerated adjoint algorithmic differentiation. Comput Phys Commun 200:300-311

36. Bultreys T, Boone MA, Boone MN, de Schryver T, Masschaele B, van Hoorebeke L, Cnudde V (2016) Fast laboratory-based micro- computed tomography for pore-scale research: Illustrative experiments and perspectives on the future. Adv Water Resour 95:341-351

37. Vinegoni C, Botnaru I, Aikawa E et al (2011) Indocyanine green enables near-infrared fluorescence imaging of lipid-rich, inflamed atherosclerotic plaques. Sci Transl Med 3:84ra45-84ra45

38. Davies-Venn CA, Angermiller B, Wilganowski N, Ghosh P, Harvey BR, Wu G, Kwon S, Aldrich MB, Sevick-Muraca EM (2012) Albumin-binding domain conjugate for near-infrared fluorescence lymphatic imaging. Mol Imaging Biol 14:301-314

39. Aikawa E, Nahrendorf M, Figueiredo J-L, Swirski FK, Shtatland T, Kohler RH, Jaffer FA, Aikawa M, Weissleder R (2007) Osteogenesis associates with inflammation in early-stage atherosclerosis evaluated by molecular imaging in vivo. Circulation 116:2841-2850

40. Yuan D, He H, Wu Y, Fan J, Cao Y (2019) Physiologically-based pharmacokinetic modeling of nanoparticles. J Pharm Sci 108:58-72

41. Kamasak ME, Bouman CA, Morris ED, Sauer K (2005) Direct reconstruction of kinetic parameter images from dynamic PET data. IEEE Trans Med Imaging 24:636-650

42. Taddio MF, Mu L, Keller C, Schibli R, Krämer SD (2018) Physiologically based pharmacokinetic modelling with dynamic PET data to study the in vivo effects of transporter inhibition on hepatobiliary clearance in mice. Contrast Media Mol Imaging 2018:1-11

43. Niederalt C, Kuepfer L, Solodenko J, Eissing T, Siegmund HU, Block M, Willmann S, Lippert J (2018) A generic whole body physiologically based pharmacokinetic model for therapeutic proteins in PKSim. J Pharmacokinet Pharmacodyn 45:235-257

44. Rosenbaum SE (2016) Introduction to physiologically based pharmacokinetic modeling. In: Rosenbaum SE (ed) Basic Pharmacokinetics and Pharmacodynamics: An Integrated Textbook and Computer Simulations, 2nd edn. Wiley, Hoboken, New Jersey, pp 373-377

Publisher's Note. Springer Nature remains neutral with regard to jurisdictional claims in published maps and institutional affiliations. 